\title{
Article
}

\section{The inverse sum indeg index (ISI) and ISI energy of Hyaluronic Acid-Paclitaxel molecules used in anticancer drugs}

\author{
Özge Çolakoğlu Havare
}

Mersin University, Science and Arts Faculty, Mathematics Department, 33343, Mersin-Turkey; ozgeeclkgl@gmail.com

Academic Editor: Roslan Hasni

Received: 17 July 2021; Accepted: 10 October 2021; Published: 24 December 2021.

\begin{abstract}
The inverse sum indeg index $\operatorname{ISI}(G)$ of a graph is equal to the sum over all edges $u v \in E(G)$ of weights $\frac{d_{u} d_{v}}{d_{u}+d_{v}}$. In this paper, we calculated the inverse indeg indices and inverse indeg energies that give information about the physicochemical properties and biological characteristics of Hyaluronic Acid-Paclitaxel conjugates used in the production of drugs used in the treatment of cancer disease. This study presents the relation between the ISI index and the ISI energy of the molecular graph of Hyaluronic Acid-Paclitaxel conjugates.
\end{abstract}

Keywords: Graph theory; Topological index; Inverse sum indeg index; Inverse sum energy; Spectral radius; Hyaluronic Acid-Paclitaxel conjugates.

MSC: 05C07; 05C50; 92E10.

\section{Introduction}

$\mathbf{L}$ et $G(V, E)$ be a simple connected graph where $V$ is vertex set and $E$ is edge set. The cardinality of the vertex set of a graph is denoted by $n$ and the cardinality of its edge set is denoted by $m$. An edge $e=u v$ of this graph connects the vertices $u$ and $v$. The degree of a vertex $u$ is denoted by $d_{u}$ and is defined in [1].

A molecular graph represents the skeletons of nonsaturated hydrocarbon of molecules and molecular compounds. The vertices and edges of this graph correspond to non-hydrogen atoms and covalent bonds between atoms, respectively. Note that hydrogen atoms are often omitted. Chemical graph theory deals with the energy levels of electrons in a molecular which is the eigenvalues of the graph and the strength of particles which is the spectrum of the graph [2,3]. Topological indices are numerical descriptors of a molecular graph. They are used to predict the physicochemical and bioactivity properties of molecules and molecular compounds [4-6].

The Wiener index which was introduced by Harold Wiener is the oldest topological index. This index was used for the physical properties of paraffin [7]. Topological indices can be classified according to the structural features of the graph. For example, the Wiener index which is based on the distance between vertices, the Randić and the Zagreb indices which are based on degree, the Estrada index which is based on the spectrum of a graph, the Hosaya index which is based on the matching, the bond-additive indices which are based measure of peripherality in graphs [4].

Hyaluronic acid (HA) which is a major component of the extracellular matrix and a naturally-occurring glycosaminoglycan is a promising molecule for use in drug production due to its biodegradable, non-toxic, hydrophilic, biocompatible, non-immutable features and unique [8]. Therefore, HA has attracted great attention in pharmaceutical production. In recent years, many scientists have shown that the use of HA in the production of anticancer drugs helps the treatment [9-11]. It has been observed that HA can be useful in the treatment of heart diseases [12]. Figure 1 shows the molecular graph of Hyaluronic acid and Figure 2 shows the molecular graph of Hyaluronic Acid-Paclitaxel (HA-PTX) conjugates with $n=3$.

Since the experiments are costly and time-consuming, it has been necessary to calculate topological indices that give information about the topology (physical chemistry and biological properties) of the molecule 


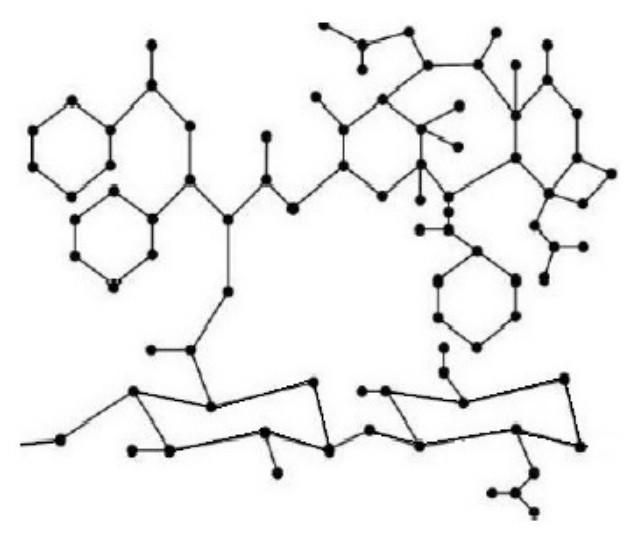

Figure 1. The molecular graph of HA-PTX[1] ([13,14]).

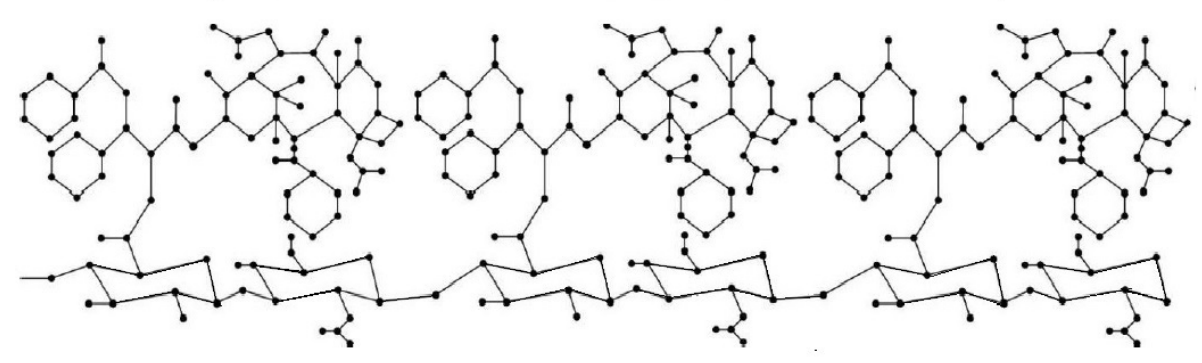

Figure 2. The molecular graph of HA-PTX[3] (see [14]).

and molecular compounds. Many scientists studied the topological indices of HA and HA-PTX conjugates $[8,13,14]$. Many studies have been conducted in chemistry and pharmaceutical science since it is useful to calculate the topological indices of molecules [15-25].

Gutman and Trinajtic defined first Zagreb index in [26] as

$$
M_{1}(G)=\sum_{u \in V(G)} d_{u}^{2}=\sum_{u v \in E(G)} d_{u}+d_{v} .
$$

Vukicevic and Gasperov introduced the discrete Adriatic indices which are bond-additive indices [27]. One of these indices is inverse sum indeg index which is defined as

$$
\operatorname{ISI}(G)=\sum_{u v \in E(G)} \frac{d_{u} d_{v}}{d_{u}+d_{v}}
$$

The inverse sum indeg adjacency matrix ISI is a matrix with entries $s_{i j}$ defined as follows [28,29]:

$$
s_{i j}=\left\{\begin{array}{ll}
\frac{d_{i} d_{j}}{d_{i}+d_{j}}, & i j \in E(G) \\
0, & \text { Otherwise }
\end{array} .\right.
$$

Let $s_{1} \geq s_{2} \geq \ldots \geq s_{n}$ be the eigenvalues of the matrix ISI. It is elementary to show that

$$
\operatorname{tr}(I S I)=\sum_{i=1}^{n} s_{i}=0
$$


where $\operatorname{tr}(I S I)$ is traces of ISI $[28,29]$. The energy of the ISI adjacency matrix is defined in $[28,29]$ as

$$
\text { ISIE }=\sum_{i=1}^{n}\left|s_{i}\right| .
$$

Lemma 1. [30,31] Let $A, B$ be Hermitian matrices of order $n$. Suppose that $A, B$ and $A+B$ be $\lambda_{i}(A), \lambda_{i}(B)$ and $\lambda_{i}(A+B)$ for $i=1,2, \ldots, n$, rexpectively, each algebraically ordered in nonincreasing order. Then, for $i=1,2, \ldots, k$,

$$
\lambda_{i}(A)+\lambda_{n}(B) \leq \lambda_{i}(A+B) \leq \lambda_{i}(A)+\lambda_{1}(B) .
$$

Vukicevic et al., found that the ISI index is an important estimator of the total surface area of octane isomers [27]. Sedlar et al., obtained extremal values for ISI index of various graph classes [32]. Lokesha et al., studied on the ISI index of some nanostructure [33]. Nezhad et al., presented the ISI index of some nanotubes [34]. Chen and Deng gave some bounds for the ISI index in terms of various graph parameters [35]. Hafeez and Farooq studied on ISI energy formula of several graph classes [36].

In this study, we computed the ISI energies, spectral radii of ISI matrices, and the ISI indices of the molecular graph of the HA-PTX conjugates. Furthermore, we compared the inverse sum indeg indices and the inverse sum indeg energies for the molecular graph of the HA-PTX conjugates used in anticancer drug production.

\section{The inverse sum indeg index (ISI), ISI energy, spectral radius of molecular graph of the Hyaluronic Acid-Paclitaxel conjugates}

Let $G$ be molecular graph of HA-PTX conjugate with $n$. Then, $\left|V\left(G_{n}\right)\right|=87 n$ and $\left|E\left(G_{n}\right)\right|=96 n-1$ $([8,13,14])$.

Theorem 1. Let $G$ be molecular graph of HA-PTX conjugate with $n=1$ unit. Then,

$$
\operatorname{ISI}(G)=\frac{6697}{60} .
$$

Proof. As discussed in $[8,13,14]$, the edge set of $G$ can be partitions as follows:

$\left|E_{1,2}\right|=\mid\left\{d_{u}=1\right.$ and $\left.d_{v}=2\right\} \mid=1$,

$\left|E_{1,3}\right|=\mid\left\{d_{u}=1\right.$ and $\left.d_{v}=3\right\} \mid=17$,

$\left|E_{1,4}\right|=\mid\left\{d_{u}=1\right.$ and $\left.d_{v}=4\right\} \mid=4$,

$\left|E_{2,2}\right|=\mid\left\{d_{u}=2\right.$ and $\left.d_{v}=2\right\} \mid=14$,

$\left|E_{2,3}\right|=\mid\left\{d_{u}=2\right.$ and $\left.d_{v}=3\right\} \mid=30$,

$\left|E_{2,4}\right|=\mid\left\{d_{u}=2\right.$ and $\left.d_{v}=4\right\} \mid=3$,

$\left|E_{3,3}\right|=\mid\left\{d_{u}=3\right.$ and $\left.d_{v}=3\right\} \mid=18$,

$\left|E_{3,4}\right|=\mid\left\{d_{u}=3\right.$ and $\left.d_{v}=4\right\} \mid=7$,

$\left|E_{4,4}\right|=\mid\left\{d_{u}=4\right.$ and $\left.d_{v}=4\right\} \mid=1$.

Assume that $\gamma_{u v}=\frac{d_{u} d_{v}}{d_{u}+d_{v}}$. From Equation (1), it can be written

$$
\begin{aligned}
I S I(G)= & \sum_{u v \in E_{1,2}} \gamma_{u v}+\sum_{u v \in E_{1,3}} \gamma_{u v}+\sum_{u v \in E_{1,4}} \gamma_{u v}+\sum_{u v \in E_{2,2}} \gamma_{u v}+\sum_{u v \in E_{2,3}} \gamma_{u v} \\
& +\sum_{u v \in E_{2,4}} \gamma_{u v}+\sum_{u v \in E_{3,3}} \gamma_{u v}+\sum_{u v \in E_{3,4}} \gamma_{u v}+\sum_{u v \in E_{4,4}} \gamma_{u v} .
\end{aligned}
$$

From Equation (4), we obtain

$$
I S I(G)=1 \times \frac{2}{3}+17 \times \frac{3}{4}+4 \times \frac{4}{5}+14 \times \frac{4}{4}+30 \times \frac{6}{5}+3 \times \frac{8}{6}+18 \times \frac{9}{6}+7 \times \frac{12}{7}+1 \times \frac{16}{8} .
$$

Theorem 2. Let $G$ be molecular graph of HA-PTX conjugate with $n=1$ unit. Then, $s_{1}(G)=7.2712$. 
Proof. The eigenvalues of the inverse sum indeg matrix of the molecular graph of the HA-PTX conjugate with $n=1$ are calculated using the MATLAB program. The positive eigenvalues are: $7.2712,4.0449,3.5368,3.2252,3.0890,3.0072,2.9687, \quad 2.6823,2.5252,2.3681,2.2741,2.1448,2.0263,1.8462$, $1.7983,1.7280,1.6222,1.6016,1.5699,1.4821,1.4599, \quad 1.2686, \quad 1.1608,1.0529,1,1,1,0.9905,0.8327,0.8027$, $0.6178,0.4489,0.3813,0.3580,0.2976,0.2290,0.2128,0.1792,0.1264$. The spectral radius of and the smallest eigenvalue of the inverse sum indeg matrix of the HA-PTX conjugate with $n=1$ are $7.2712,-7.2712$, respectively.

Theorem 3. Let EISI $(G)$ be the inverse sum energy of the molecular graph of HA-PTX[1]. Then,

$$
\operatorname{EISI}(G)=132.4624 \text {. }
$$

Proof. From Equation (2), it can be written EISI $=2 \sum_{i=1} s_{i}$ for $s_{i}>0$. The proof is complemented from the positive eigenvalues of the inverse sum indeg matrix of HA-PTX conjugate with $n=1$ in the proof of Theorem 2 and Equation (3).

Theorem 4. If $G$ is the molecular graph of HA-PTX[n] then

$$
I S I(G)=\frac{3413}{30} n-\frac{43}{20} .
$$

Proof. Assume that $G$ is the molecular graph of HA-PTX[n]. As discussed in [13], the edge set of $G$ can be partitioned as;

$$
\begin{aligned}
& \left|E_{1,2}\right|=\left|\left\{d_{u}=1, d_{v}=2\right\}\right|=n, \\
& \left|E_{1,3}\right|=\left|\left\{d_{u}=1, d_{v}=3\right\}\right|=16 n+1, \\
& \left|E_{1,4}\right|=\left|\left\{d_{u}=1, d_{v}=4\right\}\right|=4 n, \\
& \left|E_{2,2}\right|=\left|\left\{d_{u}=2, d_{v}=2\right\}\right|=13 n+1, \\
& \left|E_{2,3}\right|=\left|\left\{d_{u}=2, d_{v}=3\right\}\right|=32 n-2, \\
& \left|E_{2,4}\right|=\left|\left\{d_{u}=2, d_{v}=4\right\}\right|=3 n, \\
& \left|E_{3,3}\right|=\left|\left\{d_{u}=3, d_{v}=3\right\}\right|=19 n-1, \\
& \left|E_{3,4}\right|=\left|\left\{d_{u}=3, d_{v}=4\right\}\right|=7 n, \\
& \left|E_{4,4}\right|=\left|\left\{d_{u}=4, d_{v}=4\right\}\right|=n .
\end{aligned}
$$

Assume that $\gamma_{u v}=\frac{d_{u} d_{v}}{d_{u}+d_{v}}$. From Equation (4), the following equation is obtained

$$
\begin{aligned}
\operatorname{ISI}(G)= & n \times \frac{2}{3}+(16 n+1) \times \frac{3}{4}+4 n \times \frac{4}{5}+(13 n+1) \times \frac{4}{4}+(32 n-2) \times \frac{6}{5}+3 n \times \frac{8}{6} \\
& +(19 n-1) \times \frac{9}{6}+7 n \times \frac{12}{7}+n \times \frac{16}{8} .
\end{aligned}
$$

This proof is completed by the calculation.

Theorem 5. If $G$ is the molecular graph of HA-PTX[n], then

$$
5.9455 \leq s_{1}(G) \leq 8.5969 .
$$

Proof. Suppose that $G_{1}$ and $G$ are the molecular structures of HA-PTX conjugate with $n=1$ and HA-PTX[n], respectively. Let $I S I^{\prime}$ be the ISI matrix of $G_{1}$ which is $87 \times 87$ matrix. Let ISI be the ISI matrix of G. Figure 3 shows the bridge graph of the molecule of HA-PTX conjugates with $n=1$. By using Figure 3 , it can be written the ISI matrix of $G$ is as follows

$$
I S I=A_{87 n \times 87 n}+B_{87 n \times 87 n}
$$




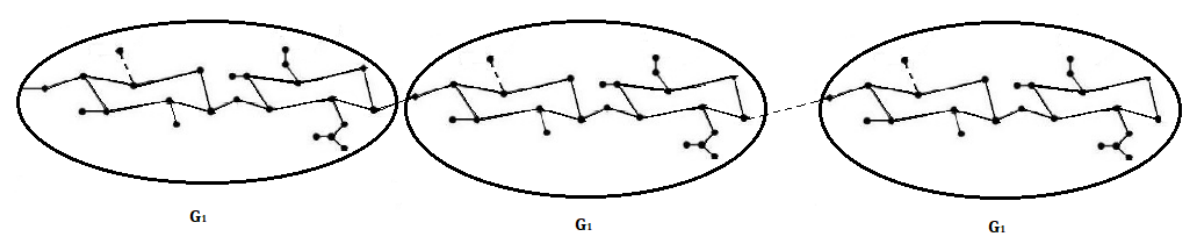

Figure 3. The bridge graph of HA-PTX conjugates with $\mathrm{n}=1$.

where

$$
A_{87 n \times 87 n}=\left[\begin{array}{cccc}
I S I^{\prime} & * & \ldots & * \\
* & I S I^{\prime} & \ldots & * \\
* & * & \ldots & * \\
* & * & \ldots & * \\
* & * & \ldots & I S I^{\prime}
\end{array}\right]_{87 n \times 87 n},
$$

where $*$ is $0_{87 \times 87}$ and

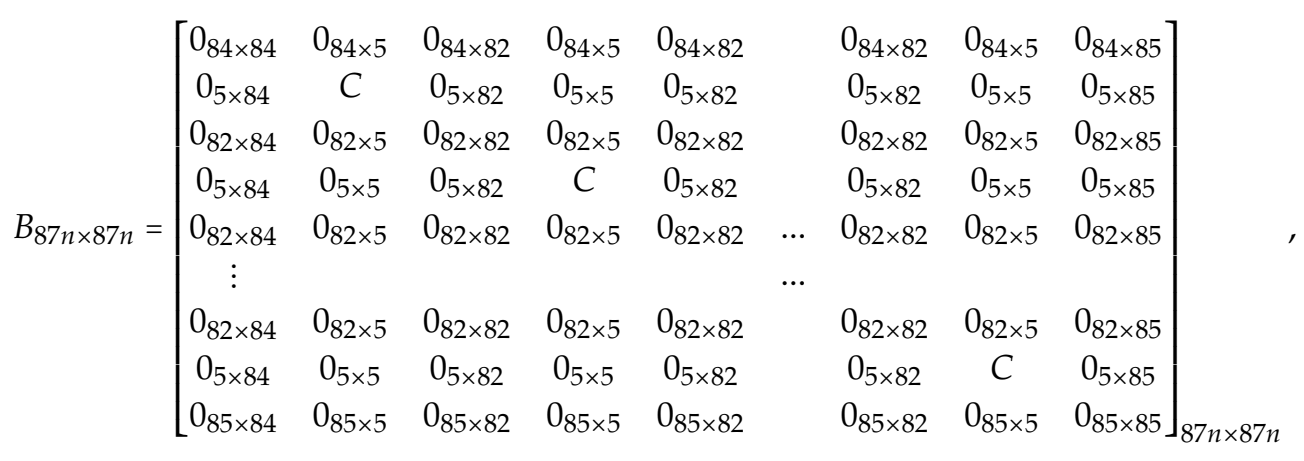

and

$$
C=\left[\begin{array}{ccccc}
0 & 1 / 5 & 0 & 0 & 0 \\
1 / 5 & 0 & 3 / 10 & 6 / 5 & 0 \\
0 & 3 / 10 & 0 & 0 & 0 \\
0 & 6 / 5 & 0 & 0 & 9 / 20 \\
0 & 0 & 0 & 9 / 20 & 0
\end{array}\right]_{5 \times 5} .
$$

By using the Matlab program, the eigenvalues of the $C$ matrix are

$$
-1.3257,-0.1224,0,0.1224,1.3257 \text {. }
$$

So, the $B$ matrix has $n-1$ eigenvalues each $-1.3257,-0.1224,0.1224$ and 1.3257 . Other eigenvalues of the $B$ matrix are 0 . The $A_{87 n \times 87 n}$ matrix has $n$ eigenvalues each the eigenvalues in the proof of Theorem 2 . From Theorem 2, the eigenvalues in (6) and Lemma 1, we have

$$
7.2712+(-1.3257) \leq s_{1}(G) \leq 7.2712+1.3257 .
$$

Theorem 6. If $G$ is the molecular graph HA-PTX $n]$, then

$$
-6.7919 n \leq \operatorname{EISI}(G) \leq 223.8799 n .
$$


Proof. From the eigenvalues in (6), it is known that the smallest eigenvalue of the $B$ matrix is -1.3257 and spectral radius of the $B$ matrix is 1.3257. By using the eigenvalues from the proof of Theorem 2 and Lemma 1 , one can obtain the eigenvalues of graph $G$ for $i=1, \ldots 87$.

$$
s_{i}(A)+s_{87 n}(B) \leq s_{i}(\operatorname{ISI}(G)) \leq s_{i}(A)+s_{1}(B) .
$$

From the above inequality, $s_{i}(\operatorname{ISI}(G))$ is negative for all eigenvalues between $(-7.2712)-(-1.4599)$ of the matrix $A$. So, the following inequality is obtained from Equation (3)

$$
\begin{gathered}
2[7.2712+\ldots+1.4599]+\sum_{i=1}^{87} s_{87 n}(B) \leq \sum_{i=1}^{87} s_{i}(I S I(G)) \leq 2[7.2712+\ldots+1.4599]+\sum_{i=1}^{87} s_{1}(B) \\
108.544-115.3359 \leq \sum_{i=1}^{87} s_{i}(I S I(G)) \leq 108.544+115.3359 \\
-6.7919 \leq \sum_{i=1}^{87} s_{i}(\operatorname{ISI}(G)) \leq 223.8799
\end{gathered}
$$

Since the $A$ matrix has $n$ eigenvalues in the proof of Theorem 2, this proof is completed.

\section{Comparison with the inverse sum indeg index, spectral radius and ISI energy of molecular graphs of Hyaluronic Acidpaclitaxel conjugates}

Table 1 shows spectral radii, the inverse sum indeg indices and the ISI energies of molecular graphs of Hyaluronic Acid-Paclitaxel (HA-PTX) conjugates with $n=1,2,3,4,5$. The eigenvalues of molecular graphs of HA-PTX conjugates with $n=2,3,4,5$ are computed by the Matlab program and the results are given in Table 2, Table 3, Table 4 and Table 5, respectively, in the appendix.

Table 1. The spectral radii, the inverse sum indeg indices and the ISI energies of Hyaluronic Acid-Paclitaxel conjugates with $n=1,2,3,4,5$.

\begin{tabular}{c|c|r|l}
$G$ & $s_{1}$ & $I S I(G)$ & $\operatorname{EISI}(G)$ \\
\hline$n=1$ & 7.2712 & 111.6166 & 132.4624 \\
\hline$n=2$ & 7.2712 & 225.3833 & 263.1968 \\
\hline$n=3$ & 7.2712 & 339.15 & 395.397 \\
\hline$n=4$ & 7.2712 & 452.9166 & 535.8942 \\
\hline$n=5$ & 7.2712 & 566.6833 & 667.5636
\end{tabular}

The graphical representation of these results was obtained with the Microsoft office excel program. Figure 4 shows a diagram of the data of Table 1. It seems that the inverse sum indeg index from the data of Table 1 and Theorem 3 is an asymptotic line. An interval for the energy is obtained in Theorem 5 when the eigenvalues for the EISI value of the Hyaluronic Acid-Paclitaxel conjugates are difficult to calculate as $n$ units increased. From this interval and the data in Table 1, we conjecture that the energy probably will be tended to line, asymptotically. Our calculations suggest that the inverse sum indeg energy of the Hyaluronic Acid-Paclitaxel conjugates can be evaluated by $\operatorname{EISI}(G)=139.29 n-3.9671$ from Table 1 . It was found that the inverse indeg energy and ISI index of the Hyaluronic Acid-Paclitaxel conjugates increase with increasing of $n$ unit.

\section{Conclusion}

Studying the physicochemical properties of a molecule or molecular compound is not only time consuming but also a costly process. Therefore, mathematical chemistry focuses on topological indices. As the number of vertices of a molecular graph increases, it is difficult to obtain data and make calculations on it. In this case, computational and statistical methods become important for analyzing the behavior of the data. In this way, the desired values can become predictable without time-consuming experiments.

HA-PTX conjugates are popular lately, especially in drug manufacturing. Therefore, it has been considered necessary to calculate the topological indices of their molecular graphs in order to examine the physicochemical and biological properties of these molecules. This study mainly aims to compare the eigenvalue-based energy of HA-PTX conjugates in the light of molecular structural analysis with the 


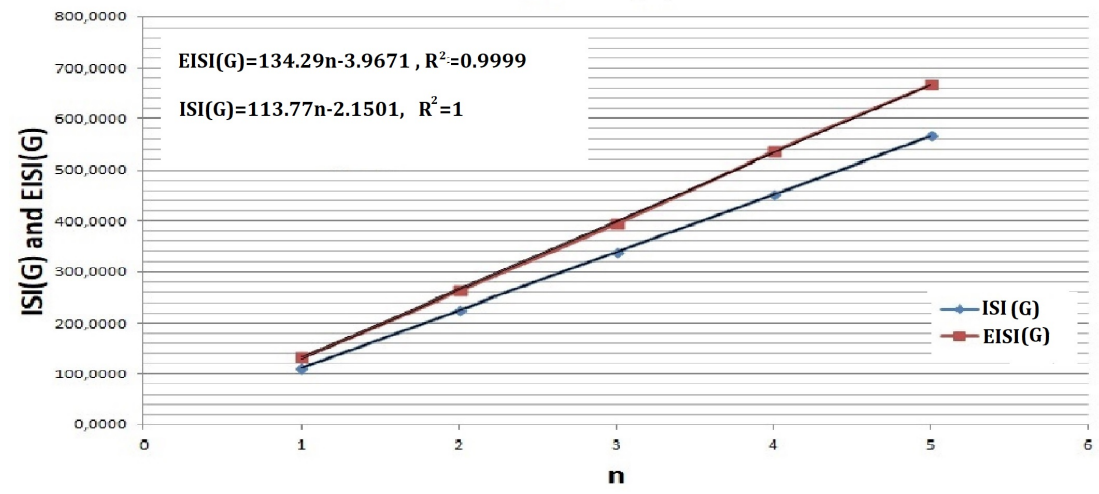

Figure 4. The diagram of ISI and EISI of malecular graphs of the Hyaluronic Acid-Paclitaxel conjugates.

degree-based index. The ISI index and ISI energy of molecular structures of HA-PTX conjugates are determined and compared. This study gives information about the energy levels of electrons and the strength of particles of the Hyaluronic Acid-paclitaxel conjugates. The results obtained in this study are thought to contribute to the applications in chemistry and pharmaceutical science in cancer treatment.

\section{Appendix}

The eigenvalues of HA-PTX conjugates with $n=2,3,4,5$ are computed by the Matlab program and the results are given in Table 2, Table 3, Table 4 and Table 5, respectively.

Table 2. The positive eigenvalues of HA-PTX conjugates with $n=2$.

$7.2712,7.2712,4.0449,4.0449,3.5368,3.5368,3.3203,3.2340,3.1426,3.0898$, $3.0384,3.0148,2.9687,2.9688,2.6823,2.6823,2.5252,2.5252,2.3740,2.3682$, $2.3034,2.2737,2.2207,2.1453,2.0642,2.0282,1.9172,1.8465,1.8384,1.7997$, $1.7283,1.7280,1.6224,1.6222,1.6179,1.1615,1.6016,1.6016,1.1605,1.5699$, $1.5699,1.4813,1.4667,1.4599,1.4310,1.2676,1.0594,1.0524,1,1,1,1,1,1$, $0.9907,0.9905,0.8445,0.8337,0.8064,0.8015,0.6180,0.6178,0.4917,0.4467$, $0.3976,0.3781,0.3583,0.3579,0.3138,0.2971,0.2926,0.2290,0.2129,0.2128$, $0.1792,0.1792,0.1272,0.1264,0.0784$

Table 3. The positive eigenvalues of HA-PTX conjugates with $n=3$.

$7.2712,7.2712,7.2712,4.0449,4.0449,4.0449,3.5368,3.5368,3.5368,3.3303$, $3.2944,3.2351,3.1612,3.1255,3.0897,3.0481,3.0286,3.0154,2.9688,2.9688$, $2.9687,2.6823,2.6823,2.6823,2.3796,2.3705,2.3683,2.3126,2.2738,2.2914$, $2.5252,2.5252,2.5252,2.2207,2.1869,2.1447,2.0888,2.0474,2.0280,1.9370$, $1.8991,1.8466,1.8391,1.8378,1.8002,1.7285,1.7283,1.7280,1.6823,1.6222$, $1.6222,1.6222,1.6016,1.6016,1.6016,1.5792,1.5699,1.5699,1.5699,1.4813$, $1.4668,1.4666,1.4599,1.4318+0.0034 i, 1.4318-0.0034 \mathrm{i}, 1.1618,1.1610,1.1605$, $1.2676,1.0524,1.0613,1.0569,1,1,1,1,1,1,1,1,1,0.9908,0.9907,0.9905$, $0.8488,0.8400,0.8353,0.8084,0.8047,0.8015,0.6181,0.6180,0.6178,0.5062$, $0.4768,0.4466,0.4011,0.3926,0.3780,0.3584,0.3582,0.3579,0.3208,0.3067$, $0.2971,0.2936,0.2912,0.2290,0.2129,0.2129,0.2128,0.1792,0.1792,0.1792$, $0.1273,0.1271,0.1264,0.0796,0.0764$ 
Table 4. The positive eigenvalues of HA-PTX conjugates with $n=4$.

\begin{tabular}{|l|}
\hline $7.2712,7.2712,7.2712,7.2712,4.0449,4.0449,4.0449,4.0449,3.5368,3.5368$, \\
$3.5368,3.5368,3.3422,3.3237,3.2818,3.2364,3.1881,3.1473,3.1200,3.0899$, \\
$3.0515,3.0406,3.0242,3.0176,2.9688,2.9688,2.9687,2.9687,2.6823,2.6823$, \\
$2.6823,2.6823,2.5252,2.5252,2.5252,2.5252,2.3833,2.3747,2.3695,2.3684$, \\
$2.3172,2.3042,2.2878,2.2738,2.2207,2.2160,2.1868,2.1453,2.0981,2.0660$, \\
$2.0403,2.0284,1.9415,1.9216,1.8852,1.8467,1.8392,1.8388,1.8371,1.8013$, \\
$1.7294,1.7283,1.7283,1.7280,1.7155,1.6249,1.6222,1.6222,1.6222,1.6217$, \\
$1.6016,1.6016,1.6016,1.6016,1.5699,1.5699,1.5699,1.5699,1.5664,1.4813$, \\
$1.4674,1.4668,1.4666,1.4599,1.4426,1.4295+0.0034 i, 1.4295-0.0034 i$, \\
$1.2683,1.1620,1.1615,1.1608,1.1605,1.0626,1.0598,1.0563,1.0524,1,1,1$, \\
$1,1,1,1,1,1,1,1,1,0.9908,0.9907,0.9906,0.9905,0.8493,0.8449,0.8380$, \\
$0.8350,0.8096,0.8064,0.8036,0.8015,0.6182,0.6178,0.6180,0.6180,0.5113$, \\
$0.4908,0.4706,0.4466,0.4018,0.3974,0.3896,0.3779,0.3584,0.3583,0.3582$, \\
$0.3579,0.3230,0.3125,0.3040,0.2970,0.2940,0.2920,0.2907,0.2290,0.2129$, \\
$0.2129,0.2129,0.2128,0.1792,0.1792,0.1792,0.1792,0.1273,0.1272,0.1271$, \\
$0.1264,0.0797,0.0770,0.0755$
\end{tabular}

Table 5. The positive eigenvalues of HA-PTX conjugates with $n=5$.

7.2712, 7.2712, 7.2712, 7.2712, 7.2712, 4.0449, 4.0449, 4.0449, 4.0449, 4.0449, $3.5368,3.5368,3.5368,3.5368,3.5368,3.3505,3.3305,3.3141,3.2759,3.2373$, $3.2040,3.1608,3.1404,3.1190,3.0899,3.0527,3.0464,3.0362,3.0222,3.0185$, $2.9687,2.9687,2.9688,2.9688,2.9688,2.6823,2.6823,2.6823,2.6823,2.6823$, $2.3859,2.3780,2.3722,2.3684,2.3691,2.3203,2.3103,2.2738,2.2971,2.2864$, 2.2207, 2.5252, 2.5252, 2.5252, 2.5252, 2.5252, 2.2158, 2.2161, 2.1868, 2.1453, $2.1011,2.0804,2.0556,2.0366,2.0287,1.9428,1.9324,1.9106,1.8749,1.8468$, $1.8392,1.8390,1.8384,1.8361,1.8021,1.7377,1.7284,1.7283,1.7283,1.7280$, $1.7264,1.6591,1.6222,1.6222,1.6222,1.6222,1.6221,1.6016,1.6016,1.6016$, $1.6016,1.6016,1.5979,1.5699,1.5699,1.5699,1.5699,1.5699,1.5610,1.4813$, $1.4675,1.4673,1.4668,1.4666,1.4599,1.4450,1.4375,1.4290+0.0028 \mathrm{i}$, 1.4290-0.0028i,1.2683,1.1623,1.1617,1.1612,1.1608,1.1605,1.0637, 1.0610,1.0585, 1.0561,1.0524,1, 1, 1, 1, 1, 1, 1, 1, 1,1,1,1,1,1,1, 0.9908,0.9908, 0.9907,0.9906, $0.9905,0.8495,0.8470,0.8419,0.8371,0.8347,0.8104,0.8075,0.8051,0.8031,0.8015$, $0.6182,0.6181,0.6180,0.6179,0.6178,0.5138,0.4996,0.4814,0.4677,0.4466$, $0.4021,0.3995,0.3944,0.3878,0.3779,0.3584,0.3584,0.3583,0.3581,0.3579$, $0.3239,0.3165,0.3084,0.3025,0.2970,0.2943,0.2927,0.2913,0.2906,0.2290$, $0.2129,0.2129,0.2129,0.2129,0.2128,0.1792,0.1792,0.1792,0.1792,0.1792$, $0.1273,0.1272,0.1271,0.1270,0.1264,0.0797,0.0774,0.0763,0.0749$

Conflicts of Interest: The author declares no conflict of interest.

Acknowledgments: The author is deeply thankful to the editor and the reviewers for their valuable suggestions to improve the quality of this manuscript.

\section{References}

[1] Lesniak, L., \& Chartrand, G. (2005). Graphs and Digraphs. London: Chapman and Hall/CRC.

[2] Chu, Z. Q., Nazeer, S., Zia, T. J., Ahmed, I., \& Shahid, S. (2019). Some new results on various graph energies of the splitting graph. Journal of Chemistry, 2019, Article ID 7214047. https://doi.org/10.1155/2019/7214047.

[3] Ghorbani, M., \& Ghazi, M. (2010). Computing some topological indices of triangular benzenoid. Digest Journal of Nanomaterials and Biostructures, 5(4), 1107-1111.

[4] Havare, O. C. (2019). QSPR Analysis with Curvilinear Regression Modeling and Topological Indices. Iranian Journal of Mathematical Chemistry, 10(4), 331-341.

[5] Shafiei, F. (2015). Relationship between topological indices and thermodynamic properties and of the monocarboxylic acids applications in QSPR. Iranian Journal of Mathematical Chemistry, 6(1), 15-28.

[6] Vukičević, D. (2011). Bond additive modeling 4. QSPR and QSAR studies of the variable adriatic indices. Croatica Chemica Acta, 84, 87-91. 
[7] Wiener, H. (1947). Structural determination of paraffin boiling points. Journal of the American Chemical Society, 69(1), 17-20.

[8] Jahanbani, A., Shao, Z., \& Sheikholeslami, S. M. (2021). Calculating degree based multiplicative topological indices of Hyaluronic Acid-Paclitaxel conjugates' molecular structure in cancer treatment. Journal of Biomolecular Structure and Dynamics, 39(14), 5304-5313.

[9] Arpicco, S., Milla, P., Stella, B., \& Dosio, F. (2014). Hyaluronic acid conjugates as vectors for the active targeting of drugs, genes and nanocomposites in cancer treatment. Molecules, 19(3), 3193-3230.

[10] Dosio, F., Arpicco, S., Stella, B., \& Fattal, E. (2016). Hyaluronic acid for anticancer drug and nucleic acid delivery. Advanced Drug Delivery Reviews, 97, 204-236.

[11] Leonelli, F., La Bella, A., Migneco, L. M., \& Bettolo, R. M. (2008). Design, synthesis and applications of hyaluronic acid-paclitaxel bioconjugates. Molecules, 13(2), 360-378.

[12] Yoon, S. J., Fang, Y. H., Lim, C. H., Kim, B. S., Son, H. S., Park, Y., \& Sun, K. (2009). Regeneration of ischemic heart using hyaluronic acid-based injectable hydrogel. Journal of Biomedical Materials Research Part B: Applied Biomaterials, 91(1), 163-171.

[13] Wang, J., Wang, Y., Wang, Y., \& Zheng, L. (2020). Computation on the topological indices of hyaluronic acid. Journal of Applied Analysis E Computation, 10(3), 1193-1198.

[14] Zheng, L., Wang, Y., \& Gao, W. (2019). Topological Indices of Hyaluronic Acid-Paclitaxel ConjugatesŠ Molecular Structure in Cancer Treatment. Open Chemistry, 17(1), 81-87.

[15] Imran, M., Bokhary, S. A. U. H., Manzoor, S., \& Siddiqui, M. K. (2020). On molecular topological descriptors of certain families of nanostar dendrimers. Eurasian Chemical Communications, 2(6), 680-687.

[16] Ashrafi, A. R. (2010). Experimental results on the energy and Estrada index of HC5C7 [4p, 8] nanotubes. Optoelectronics and Advanced Materials-Rapid Communications, 4(January 2010), 48-49.

[17] Ahmad, Z., Mufti, Z. S., Nadeem, M. F., Shaker, H., \& Siddiqui, H. M. A. (2021). Theoretical study of energy, inertia and nullity of phenylene and anthracene. Open Chemistry, 19(1), 541-547.

[18] Nadeem, M. F., Imran, M., Siddiqui, H. M. A., Azeem, M., Khalil, A., \& Ali, Y. (2021). Topological aspects of metal-organic structure with the help of underlying networks. Arabian Journal of Chemistry, 14(6), 103157. https://doi.org/10.1016/j.arabjc.2021.103157.

[19] Bakhshi, K., \& Mojallal, S. A. (2013). Relation between topological indices and exchange-correlation energy for Graphene Nanosurface: A DFT study. Fullerenes, Nanotubes and Carbon Nanostructures, 21(7), 617-623.

[20] Malik, M. A., \& Farooq, R. (2015). Computational results on the energy and Estrada index of TUC4C8 (R)[m, n] nanotubes. Optoelectronics and Advanced Materials-Rapid Communications, 9(1-2), 311-313.

[21] Hayat, S., Imran, M., \& Liu, J. B. (2019). Correlation between the Estrada index and $\pi$-electronic energies for benzenoid hydrocarbons with applications to boron nanotubes. International Journal of Quantum Chemistry, 119(23), e26016.

[22] Kulli, V. R. (2019). On hyper KV and square KV indices and their polynomials of certain families of dendrimers. Journal of Computer and Mathematical Sciences, 10(2), 279-286.

[23] Ediz, S. (2013). The Ediz eccentric connectivity index of one pentagonal carbon nanocones. Fullerenes, Nanotubes and Carbon Nanostructures, 21(2), 113-116.

[24] Nadeem, M. F., Azeem, M., \& Farman, I. (2021). Comparative study of topological indices for Capped and Uncapped Carbon Nanotubes. Polycyclic Aromatic Compounds, https:/ /doi.org/10.1080/10406638.2021.1890625.

[25] Koorepazan-Moftakhar, F., Ori, O., \& Ashrafi, A. R. (2019). Symmetry-based invariants of nanostructures and their effect on edge states of carbon nanotubes. Fullerenes, Nanotubes and Carbon Nanostructures, 27(3), 215-224.

[26] Gutman, I., \& Trinajstić, N. (1972). Graph theory and molecular orbitals. Total $\phi$-electron energy of alternant hydrocarbons. Chemical Physics Letters, 17(4), 535-538.

[27] Vukičević, D., \& Gašperov, M. (2010). Bond additive modeling 1. Adriatic indices. Croata Chemica Acta, 83, $243-260$.

[28] Das, K. C., Gutman, I., Milovanović, I., Milovanović, E., Furtula, B. (2018). Degree-based energies of graphs. Linear Algebra and its Applications, 554, 185-204.

[29] Zangi, S., Ghorbani, M., \& Eslampour, M. (2018). On the eigenvalues of some matrices based on vertex Degree. Iranian Journal of Mathematical Chemistry, 9(2), 149-156.

[30] Guo, J. M., Wang, Z. W., \& Li, X. (2019). Sharp upper bounds of the spectral radius of a graph. Discrete Mathematics, 342(9), 2559-2563.

[31] Horn R. A., \& Johnson, C. R. (2013). Matrix Analysis, 2nd ed. Cambridge University Press.

[32] Sedlar, J., Stevanović, D., \& Vasilyev, A. (2015). On the inverse sum indeg index. Discrete Applied Mathematics, 184, 202-212. 
[33] Lokesha, V., Deepika, T., \& Cangul, I. N. (2017). Symmetric division deg and inverse sum indeg indices of Polycyclic Aromatic Hydrocarbons (PAHs) and Polyhex Nanotubes. Southeast Asian Bulletin of Mathematics, 41(5), 707-715.

[34] Nezhad, F. F., \& Azari, M. (2016). The inverse sum indeg index of some nanotubes. Studia Universitatis Babes-Bolyai, Chemia, 61(1), 63-70.

[35] Chen, H., \& Deng, H. (2018). The inverse sum indeg index of graphs with some given parameters. Discrete Mathematics, Algorithms and Applications, 10(1), 1850006. https://doi.org/10.1142/S1793830918500064.

[36] Hafeez, S., \& Farooq, R. (2019). Inverse sum indeg energy of graphs. IEEE Access, 7, 100860-100866.

( 2021 by the authors; licensee PSRP, Lahore, Pakistan. This article is an open access article distributed under the terms and conditions of the Creative Commons Attribution (CC-BY) license (http://creativecommons.org/licenses/by/4.0/). 